\title{
THEORY OF GRAVITY INSTABILITY WITH VARIABLE OVERBURDEN AND COMPACTION
}

BY

M. A. BIOT AND H. ODE

Reprinted for private circulation from Geopirysics

Vol. XXX, No. 2, April, 1965 


\title{
THEORY OF GRAVITY INSTABILITY WITH VARIABLE OVERBURDEN AND COMPACTION $\dagger$
}

\author{
M. A. BIOT* AND H. ODE
}

The gravitational instability of a simple two-layered medium resting on a rigid base has been treated in terms of the analog model of Biot. In order to avoid complications of purely mathematical nature which are unrelated to the physics of the process of instability and do not affect the results significantly, the analysis is presented for the two-dimensional case. ${ }^{1}$ It is shown that such a system is unstable if the density contrast $\Delta \rho$ between the top layer (overburden) and the bottom layer (salt) is positive. The physically important parameters of the instability are the viscosity ratio and the thickness ratio between overburden and salt and the relative density contrast $\Delta \rho / \rho_{1}$. These parameters determine the amplification rate of a given initial sinusoidal perturbation of wavelength $L$ of the overburden-salt interface. For the purpose of isolating the role of each parameter and to provide an understanding of the underlying mechanics, several tables are presented which show the influence of each of these parameters as well as the wavelength of maximum amplification rate (dominant wavelength) and the time in which a thousandfold amplification is obtained (characteristic time). This form of presentation of results is well suited to geological problems including the variable time dependence of the overburden due to gradual sedimentation. The influence of either an additional surface layer of water or a nonrigid base of high viscosity is small. The special condition of keeping the surface of the upper layer flat while the volume is held constant, termed "redistribution," leads to an increased rate of growth and slightly longer dominant wavelength.

The geologically more significant case of an instability with time-dependent thickness and compaction of overburden is solved by introduction of an effective density $\rho_{e}$ of the overburden. The characteristic times and dominant wavelengths of salt structures in a salt layer of initial thickness of $1,000 \mathrm{~m}$ and viscosity of $10^{17}$ poises overlain by sediment of viscosity of $10^{20}$ poises are in excellent agreement with observed facts.

\section{INTRODUCTION}

It is now generally accepted that many of the large salt structures discovered so far in several areas of the world owe their existence to the upward driving force of buoyancy. The first to propose this theory was Arrhenius (1912), but it was not until 1934, when Nettleton demonstrated the mechanism convincingly with a model of viscous liquids of contrasting color and different densities, that it became widely accepted. The scaling factor of this viscous model was shown to correspond to the approximate salt viscosity and to the geological time scale (Hubbert, 1937). Recently, experiments with tar and heavy mud (Parker and McDowell, 1955), whose properties were chosen to obtain some degree of dynamical similitude, have shown the correctness of Arrhenius's hypothesis.
So far little analytical work has been done to estimate the geometry and the rate of deformation of the interface between the two media. In the present paper a simple theory of the instability of a two-layered medium resting on a rigid base is presented. It is an application of the stability theory for a medium consisting of viscoelastic layers, which was recently published by Biot (1963a, b), and contains preliminary numerical solutions for salt-structure instability. In the latter paper it was shown that the formulation of the problem is greatly simplified by replacing the layered medium by an analog model in which gravity is accounted for by surface forces acting at the free surface and at the interfaces between layers. An outline of the method is given in Section 2. In the general stability equation for the two-layered model appear two operators $\hat{Q}_{1}$ and

$\dagger$ EPR Publication No. 377, Shell Development Company (A Division of Shell Oil Company), Exploration and Production Research Division, Houston, Texas. Manuscript received by the Editor June 22, 1964 .

* Consultant, Shell Development Company, New York.

† Shell Development Company, Houston, Texas.

${ }^{1}$ Comments were received by the authors from M. King Hubbert regarding the essentially three-dimensional character of salt structures. It turns out that only a slight quantitative correction must be introduced when applying two-dimensional solutions to the three-dimensional case. This extension of the theory will be discussed in a forthcoming paper (M. A. Biot, "Three-dimensional gravity instability derived from two-dimensional solutions"). 
$\hat{Q}_{2}$, which, if we are dealing with purely viscous materials, represent the expressions $\eta_{1} p$ and $\eta_{2} p$, $\eta_{1}$ and $\eta_{2}$ being the viscosities of the two layers, respectively, and $p=d / d t$. The rate of growth of an initially present sinusoidal perturbation of the interface is amplified with an exponential rate $e^{p t}$.

The results of the present theory can be applied immediately to the problem of salt tectonics; of course, the theory applies equally well to other materials with appropriate properties. The top layer in our model represents the layer of sediments (overburden) overlying the second layer, which represents the salt. The assumption that salt and overburden can be regarded as Newtonian fluids is questionable. At best it is possible to ascribe to these materials only an "equivalent" viscusity, which will be a function of confining pressure, differential load, temperature, presence of liquids, etc. For salt an approximate value of an equivalent viscosity can be obtuined from measurements on the steady-state creep. A survey of available data has been made (Odé, 1962), and a reasonable estimate for the equivalent salt viscosity appears to be $10^{17}$ poises. The information concerning its values for materials constituting the overburden is exceedingly flimsy, and indeed trustworthy data are nonexistent. Nevertheless we have tentatively assumed that the equivalent viscosity of the overburden is about two or three orders of magnitude larger than that of salt, and we have adopted a value of $10^{20}$ poises.

The stability analysis for the two-layered structure is worked out in Section 3. It is found that the resulting stability equation is quadratic and has a positive root if the density of the top layer exceeds that of the second layer. This shows that in that case there is instability. Of particular interest is the time $t_{c}$ in which the amplitude of an originally present perturbation of the interface is increased one thousand times. We have called this the characteristic time, which is useful for expressing the rate of amplification. The rate at which an initially present perturbation of wavelength $L$ is amplified is found to be a function of three parameters: the viscosity ratio $\eta_{1} / \eta_{2}$, the thickness ratio $h_{1} / h_{2}$, and the relative density contrast $\left(\rho_{1}-\rho_{2}\right) / \rho_{1}$, the subscripts 1 and 2 indicating upper and lower layer, respectively. The influence of each of these parameters on the rates of amplification is shown in several tables. For the original thickness of the salt we have adopted the value of $10^{5} \mathrm{~cm}$. This value appears to be of the right order of magnitude for the northwest European and Gulf Coast basins.

For completeness, a brief analysis is included of the influence of water on top of the system and of the presence of a nonrigid but viscous base.

In Section 4 we consider the instability with the additional complication that the upper surface is kept flat by various geologic processes such as wave action or erosion. High spots are supposed to be leveled, and the eroded materials are supposed to be deposited in the low areas. This process we term "redistribution of surface material." Its effect is to introduce nonuniform loading at the upper boundary of the top layer, which speeds up the instability.

The assumption that the three parameters remain constant during the development of the instability is geologically unrealistic. Sediments slowly build up an overburden of increasing thickness over the salt layer and increase in density by compaction. First there is a period in which the density of the overburden is less than that of the salt, but after sufficient time the density of the overburden reaches and then exceeds the salt density. Given the rate of sedimentation and compaction, it is possible to estimate the characteristic time of any wavelength. In Section 5 this estimate has been made. Here the advantage of the analog model representation is clearly demonstrated by the introduction of an effective density $\rho_{e}(t)$ for the overburden.

\section{GENERAL STABILITY EQUATIONS}

General equations for the stability of a layered continuum under initial stress have been derived in previous work (Biot, 1963a, b). For a medium composed of horizontal layers of incompressible materials, where the initial stress is reduced to a hydrostatic field in equilibrium with the gravity, forces, the problem lcads to a simplified formulation as follows.

It was found that the system is equivalent to an analog model which is weightless. In this model gravity has been replaced by surface forces acting at the free surface and at the interfaces between layers. These surface forces are analogous to elastic forces proportional to the vertical displacement and to the density discontinuity.

The problem can then be formulated by first considering a weightless layer of thickness $h$. The 


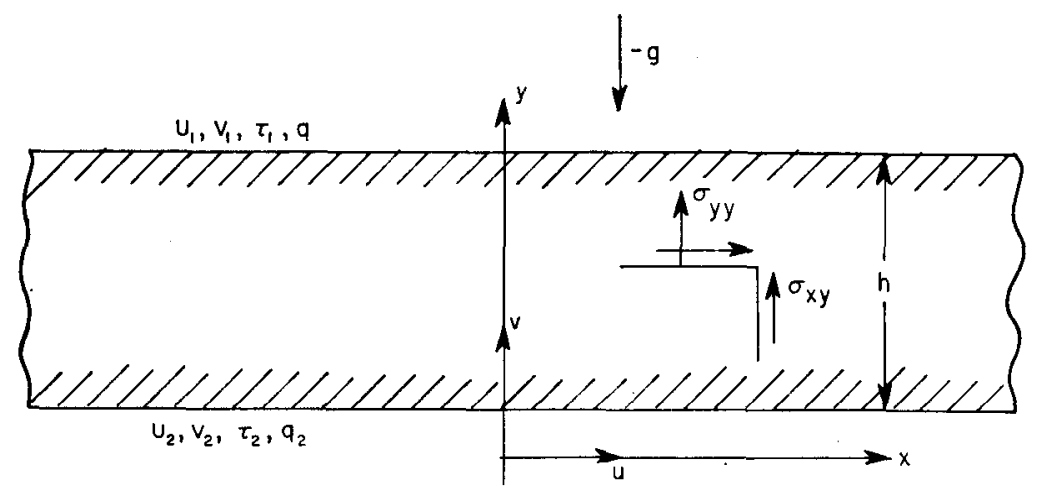

FIG. 1. Coordinates and variables for the single layer.

$y$ axis is chosen vertical and normal to the layer. The deformation is assumed to be parallel with the $x, y$ plane and to be sinusoidal along $x$ (Figure 1).

The $x$ and $y$ components of the displacements can therefore be written

$$
\begin{aligned}
& u=U \sin l x, \\
& v=V \cos l x,
\end{aligned}
$$

where $U$ and $V$ are functions of $y$. The tangential and normal components of the stress on a plane normal to the $y$ axis arising from the incremental forces acting per unit initial area at the boundary can similarly be written

$$
\begin{aligned}
& \sigma_{x y}=\tau \sin l x, \\
& \sigma_{y y}=q \cos l x .
\end{aligned}
$$

We denote by $U_{1}, V_{1}, \tau_{1}$, and $q_{1}$ the values of $U$, $V, \tau$, and $q$ at the top of the layer and by $U_{2}, V_{2}$, $\tau_{2}$, and $q_{2}$ the values of the same variables at the bottom. Thus $\tau_{1}$ and $q_{1}$ are the stress amplitudes at the top of the layer, and $\tau_{2}$ and $q_{2}$ are the stress amplitudes at the bottom of the layer.

It is a simple problem to derive the relation between the stress components applied at the two faces and the normal and tangential displacements at these faces. If the medium is assumed to be isotropic, the solution is formally the same as in the classical theory of elasticity. of isotropic media. Since we also assume incompressibility, the solution contains a single elastic coefficient, namely, the shear modulus $\mu$. For a viscoelastic medium the solution is formally identical, except that the shear modulus is replaced by an operator
$\hat{Q}$ representing the viscoelastic stress-strain relations.

The result is obtained in the following form:

$\left[\begin{array}{l}\tau_{1} \\ q_{1} \\ \tau_{2} \\ q_{2}\end{array}\right]$

$=\operatorname{lQ}\left[\begin{array}{rrrr}A & B & -D & E \\ B & C & -E & F \\ D & E & -A & B \\ -E & -F & B & -C\end{array}\right]\left[\begin{array}{l}U_{1} \\ V_{1} \\ U_{2} \\ V_{2}\end{array}\right]$.

The matrix coefficients are

$A=\frac{1}{2}\left(a_{11}+b_{11}\right), \quad D=\frac{1}{2}\left(a_{11}-b_{11}\right)$,

$B=\frac{1}{2}\left(a_{12}+b_{12}\right), \quad E=\frac{1}{2}\left(a_{12}-b_{12}\right)$,

$C=\frac{1}{2}\left(a_{22}+b_{22}\right), \quad F=\frac{1}{2}\left(a_{22}-b_{22}\right)$.

The six distinct quantities $a_{i j}$ and $b_{i j}$ in these coefficients are functions only of

$$
\gamma=\frac{1}{2} l h=\frac{\pi h}{L} .
$$

The parameter $\gamma$ is inversely proportional to the ratio of the wavelength $L$ to the thickness $h$ of the layer. The six functions $a_{i j}$ and $b_{i j}$ are 


$$
\begin{array}{ll}
a_{11}=\frac{4 \cosh ^{2} \gamma}{\sinh 2 \gamma+2 \gamma}, & b_{11}=\frac{4 \sinh ^{2} \gamma}{\sinh 2 \gamma-2 \gamma}, \\
a_{12}=-\frac{4 \gamma}{\sinh 2 \gamma+2 \gamma}, & b_{12}=\frac{4 \gamma}{\sinh 2 \gamma-2 \gamma}, \\
a_{22}=\frac{4 \sinh ^{2} \gamma}{\sinh 2 \gamma+2 \gamma}, & b_{22}=\frac{4 \cosh ^{2} \gamma}{\sinh 2 \gamma-2 \gamma} .
\end{array}
$$

Until now the layer has been assumed weightless. Let us introduce a gravity field of acceleration $g$ acting perpendicularly to the layer in the negative $y$ direction. The mass density of the layer is assumed to be constant and is denoted by $\rho$.

It was shown (Biot, 1963b) that the effect of gravity can be introduced quite simply by using the result for the weightless case. The initial stress is assumed to be the same as that in a fluid in equilibrium with the gravity field and with the same density distribution as the medium. On a horizontal plane the initial tangential stress is zero, and the normal component is the negative pressure of the initial hydrostatic field. After deformation the horizontal plane becomes a corrugated surface with sinusoidal amplitude. We denote by $T_{x y}$ and $T_{v v}$ the tangential and normal stress increments on a deformed surface which is a horizontal plane in the initial state. The values of these increments were shown to be (Biot, 1963a)

$$
\begin{aligned}
& T_{x y}=\tau \sin l x, \\
& T_{y y}=(q+\rho g V) \cos l x,
\end{aligned}
$$

where $\tau$ and $q$ are the same as evaluated for the weightless case by equation (3), and $\rho$ and $V$ are the density of the plate and the vertical displacement amplitude of the interface considered, respectively.

Consider now the condition at the interface between two layers. Let us designate by prime quantities the variables in the bottom layer. Since the normal and tangential stress increments acting along the interface are continuous at any moment, we can write

$$
\begin{aligned}
\tau_{2} & =\tau_{1}^{\prime}, \\
q_{2}+\rho g V & =q_{1}^{\prime}+\rho^{\prime} g V .
\end{aligned}
$$

The second equation of (8) is also written

$$
q_{2}=q_{1}^{\prime}+\left(\rho^{\prime}-\rho\right) g V .
$$

This shows that the effect of gravity is analogous to the weightless case with the addition of an interfacial force of magnitude $\left(\rho^{\prime}-\rho\right) g V$ per unit area and acting positively downward. If the bottom layer is denser $\left(\rho^{\prime}>\rho\right)$, an upward displacement $V$ produces a downward force analogous to an elastic restoring force and hence is stabilizing. If the top layer is denser, the surface force is upward and thus is destabilizing.

As already mentioned, $\hat{Q}$ denotes an operator which defines the viscoelastic properties of the layer. There are two extreme cases of particular interest. For a purely elastic medium the operator is identical with the shear modulus

$$
\hat{Q}=\mu \text {. }
$$

If the layer is purely viscous of Newtonian viscosity $\eta$, we put

$$
\hat{Q}=\eta p,
$$

where $p$ denotes the operation of differentiation with respect to time,

$$
p=\frac{d}{d t} .
$$

In that case the right-hand sidc of cquation (3) is expressed in terms of first-order time derivatives of the displacement amplitudes $U_{i}, V_{i}$.

In stability problems involving viscoclastic layers we are seeking solutions proportional to an exponential factor $\exp (p t)$. In this case the symbol $p$ in the operator may be treated as an algcbraic quantity representing the coefficient $p$ in the exponent.

\section{STABILITY ANALYSIS OF A TWO- LAYERED STRUCTURE}

We shall consider the two-layered horizontal structure represented in Figure 2. The material is assumed to be purely viscous. The bottom layer of thickness $h_{2}$, density $\rho_{2}$, and viscosity $\eta_{2}$ lies with perfect adherence on a rigid horizontal base. The top layer of thickness $h_{1}$ has a viscosity $\eta_{1}$ and a density $\rho_{1}$ greater than $\rho_{2}$. The materials are incompressible and under the action of a uniform gravity field $g$.

Geologically this may be looked upon as a simplified model representing a salt layer of density $\rho_{2}$ and an overburden of density $\rho_{1}$.

We shall derive the stability equations for this system assuming a plane strain deformation with 


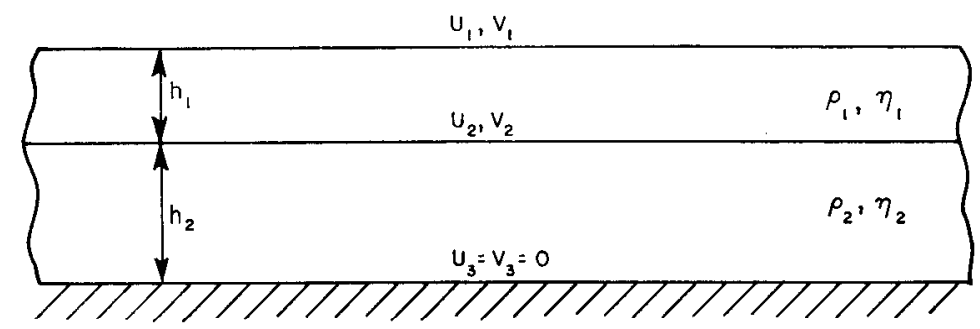

FIG. 2. Two-layered structure resting on a rigid base.

a displacement field in a vertical plane $(x, y)$. We denote by $U_{1}, V_{1}$ the displacement of the top layer at the free surfacc. The common displacement of the two adhering layers at the interface is denoted by $U_{2}, V_{2}$. The bottom displacement of the lower layer is zero, since the lower layer is assumed to adhere to a rigid base.

The stability equations are obtained by expressing that the stresses vanish at the free surface $\left(T_{x y}=T_{y y}=0\right)$, that they are continuous at the interface [equation (8)], and that the displacements vanish along the rigid base $\left(U_{3}=V_{3}=0\right)$. Applying the general equations (3) of the previous section and equations (8), we obtain

$$
\begin{aligned}
& 0=l \hat{Q}_{1}\left(A_{1} U_{1}+B_{1} V_{1}-D_{1} U_{2}+E_{1} V_{2}\right) \\
& 0=l \hat{Q}_{1}\left(B_{1} U_{1}+C_{1} V_{1}-E_{1} U_{2}+F_{1} V_{2}+\rho_{1} g V_{1}\right. \\
& l \hat{Q}_{2}\left(A_{2} U_{2}+B_{2} V_{2}\right) \\
& \quad=l \hat{Q}_{1}\left(D_{1} U_{1}+E_{1} V_{1}-A_{1} U_{2}+B_{1} V_{2}\right) \\
& l \hat{Q}_{2}\left(B_{2} U_{2}+C_{2} V_{2}\right) \\
& \quad=l \hat{Q}_{1}\left(-E_{1} U_{1}-F_{1} V_{1}+B_{1} U_{2}-C_{1} V_{2}\right) \\
& \quad+\left(\rho_{1}-\rho_{2}\right) g V_{2} .
\end{aligned}
$$

We put, according to equation (10),

$$
\begin{aligned}
& \hat{Q}_{1}=\eta_{1} p \\
& \hat{Q}_{2}=\eta_{2} p .
\end{aligned}
$$

The coefficients $A_{1}, B_{1}$, etc., are obtained from equations (4) and ( 6 ) in which $\gamma$ is replaced by

$$
\gamma_{1}=\frac{1}{2} l h_{1} .
$$

They are functions only of $\gamma_{1}$. Similarly $A_{2}, B_{2}, C_{2}$ are functions only of

$$
\gamma_{2}=\frac{1}{2} l h_{2} .
$$

The stability problem is solved by equating to zero the determinant of equations (11). This characteristic equation is written in the form

$$
a \sigma^{2}+b \sigma+c=0,
$$

where $a, b$, and $c$ are functions of the following nondimensional parameters:

$$
\begin{aligned}
& \frac{\eta_{1}}{\eta_{2}}=\text { viscosity ratio, } \\
& \frac{h_{1}}{h_{2}}=\text { thickness ratio, } \\
& \frac{\Delta \rho}{\rho_{1}}=\frac{\rho_{1}-\rho_{2}}{\rho_{1}}=\text { relative density contrast, } \\
& \gamma_{2}=\frac{1}{2} h_{2} l .
\end{aligned}
$$

The unknown $p$ is contained in the parameter

$$
\sigma=\frac{\left(\rho_{1}-\rho_{2}\right) g h_{2}}{\eta_{1} p} .
$$

By a previously proved (Biot, 1963b) theorem, the roots $p$ are real; hence $\sigma$ is also real. For given values of the parameters $\eta_{1} / \eta_{2}, h_{1} / h_{2}$, and $\Delta \rho / \rho_{1}$, the variable $\sigma$ can be plotted as a function of $\gamma_{2}$. Note that according to equation (5), $\gamma_{2}$ is proportional to the ratio of the thickness $h_{2}$ to the wavelength $L$. Such a plot therefore represents in nondimensional form a relation between $p$ and the wavelength. For each wavelength $L=\pi h_{2} / \gamma_{2}$ there is a sinusoidal solution along the horizontal direction in which the amplitude increases exponentially with time in proportion to the factor $\exp (p t)$. The stability equation (15) is quadratic and therefore has two roots. A negative root means that the amplitude of an initially present perturbation of the interface is damped out in time and thus will 
disappear. In contrast, a positive root signifies that the amplitude will grow and thus is the significant one in the evaluation of the interfacial instability. In the present case of two layers we find that one root is positive and one root is negative. In the following discussion we shall denote the positive root by $\sigma$. In general it can be shown that in a multilayered system the number of roots is equal to the number of nonrigid interfaces, including the top surface.

The positive root $\sigma$ is a function of $p$, and the coefficients $a, b$, and $c$ of equation (15) are functions of $\gamma_{2}$. Hence a graph of $\sigma$ versus $\gamma_{2}$ will show the rate of growth of given wavelengths. For very small and very large wavelengths the rate of growth of the amplitude is vanishingly small $(\sigma \rightarrow \infty)$, but for a certain wavelength it reaches a maximum, or in other words, $\sigma$ has a minimum. This wavelength is called "dominant" (Biot, 1961) because it will stand out more and more clearly as deformation proceeds. Although any wavelength of an initially present irregularity of the interface is being amplified at any moment, the dominant wavelength grows so fast that its amplitude becomes greater and greater with respect to the amplitudes of all others. Thus, in an initial perturbation of arbitrary shape the dominant wavelength will become the wavelength finally observed, and this is the reason it is called "dominant." We denote it by $L_{d}$.

As a measure of the rate of growth of the dominant wavelength a "characteristic time" $t_{c}$ is introduced. This is the time in which the original amplitude of the dominant wavelength at $t=0$ is amplified one thousand times. The exponential factor of amplification

$$
A=e^{p t}
$$

is called the amplification factor by Biot (1961). By equations (17) and (18), putting $A=10^{3}$, we have for the characteristic time

$$
t_{c}=\frac{3 \eta_{1} \sigma_{\min }(\ln 10)}{\left(\rho_{1}-\rho_{2}\right) g h_{2}} .
$$

The minimum value of $\sigma$, when plotted against $\gamma_{2}$, is denoted by $\sigma_{\min }$.

Given the values of $\eta_{1}, \eta_{2}, h_{1}, h_{2}, \rho_{1}$, and $\rho_{2}$, it is possible to compute $L_{d}$ and $t_{c}$. In the present discussion the following values have been chosen for the second layer:

$$
\begin{gathered}
\eta_{2}=10^{17} \text { poises; } \quad h_{2}=10^{5} \mathrm{~cm} ; \\
\rho_{2}=2.2 \mathrm{~g} / \mathrm{cm}^{3} .
\end{gathered}
$$

This second layer thus represents a layer of salt of $1,000-\mathrm{m}$ thickness. The properties of the top layer are specified by means of the parameters (16). To assess the influence of a change in these parameters on $L_{d}$ and $t_{c}$, three tables are presented, each of which is computed by varying one of the parameters only and keeping the other two constant.

\section{a) Dependence on the viscosity ratio $\eta_{1} / \eta_{2}$}

In Table 1 the ratio $L_{d} / h_{2}$ and the time $t_{c}$, expressed in years, are tabulated for varying $\eta_{1} / \eta_{2}$, with the values in equation (20) used for the physical constants.

\begin{tabular}{rcl}
\multicolumn{4}{c}{ Table 1 } \\
& Case $\frac{h_{1}}{h_{2}}=1 ; \quad \frac{\Delta_{\rho}}{\rho_{1}}=0.1$ \\
\hline$\eta_{1} / \eta_{2}$ & $L_{d} / h_{2}$ & \multicolumn{1}{c}{$t_{c}$ (years) } \\
\hline 1 & 3.14 & $0.0104 \times 10^{6}$ \\
10 & 3.95 & $0.046 \times 10^{6}$ \\
100 & 5.71 & $0.36 \times 10^{6}$ \\
1,000 & 8.72 & $3.36 \times 10^{6}$ \\
\hline
\end{tabular}

In Figure 3 we have shown the curves $\sigma$ versus $\gamma_{2}$ for $\eta_{1} / \eta_{2}=1,10,100$, and 1,000 , with $h_{1} / h_{2}=1$, and $\Delta \rho / \rho_{1}=0.1$. These results mean that when the viscosity of the upper layer is increased $\left(\eta_{2}\right.$ is considered fixed), the dominant wavelength increases, and the rate of amplification decreases.

\section{b) Dependence on the thickness ratio}

In Table 2 the ratio $L_{d} / h_{2}$ and the time $t_{c}$, expressed in years, are tabulated for varying $h_{1} / h_{2}$, with $\eta_{1} / \eta_{2}=1,000$, and $\Delta \rho / \rho_{1}=0.1$; the values in equation (20) are used for the physical constants.

The curves $\sigma$ versus $\gamma_{2}$ for this case are plotted in Figure 4. The case $h_{1} / h_{2}=\infty$ corresponds to an infinite overburden. This case was discussed in more detail by Biot (1963b). It is also obtained from equations (11) by putting $\gamma_{1}=\infty$, which results in considerable simplification and a linear equation for $\sigma$. A further discussion of the case of the infinite overburden will be included at the end of this section.

Examination of Table 2 and Figure 4 shows 


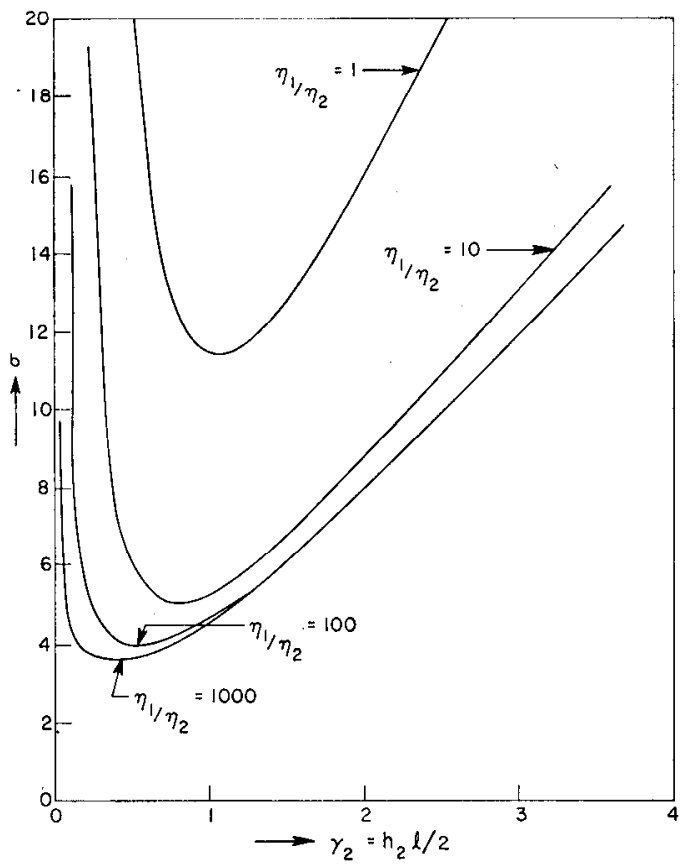

FIG. 3. $\sigma$ versus $\gamma_{2}$ for $h_{1} / h_{2}=1, \Delta \rho / \rho_{1}=0.1$, and varying viscosity ratio.

clearly that for a thin top layer the short wavelengths are more strongly amplified than the longer ones. For indefinitely large thickness of the upper layer, the dominant wavelength tends to a limiting finite value. However, when the upper layer is thin, the characteristic time $t_{c}$ becomes very large, indicating that the deformation takes

Table 2

$$
\text { Case } \frac{\eta_{1}}{\eta_{2}}=1,000 ; \quad \frac{\Delta \rho}{\rho_{1}}=0.1
$$

\begin{tabular}{ccc}
\hline$h_{1} / h_{2}$ & $L_{d} / h_{2}$ & \multicolumn{1}{c}{$t_{c}$ (years) } \\
\hline 0.1 & 1.61 & $32.92 \times 10^{6}$ \\
1.0 & 8.72 & $3.36 \times 10^{6}$ \\
5 & 19.63 & $0.87 \times 10^{6}$ \\
20 & 27.32 & $0.63 \times 10^{6}$ \\
$\infty$ & 27.6 & $0.63 \times 10^{6}$ \\
\hline
\end{tabular}

a long time to build up. The plot of $\sigma$ versus $\gamma_{2}$ also shows that the minimum of $\sigma$ is very flat for the case of a thin overburden (see case $h_{1} / h_{2}=0.1$ of Figure 4). The rate of emergence of the dominant wavelength from a randomly perturbed interface is expressed by the selectivity; this has been discussed by Biot (1961). The fatness of the minimum of $\sigma$ shows a lack of selectivity for a thin top layer.

Of particular interest is the rapid decrease of the characteristic time $t_{c}$ for $h_{1}>h_{2}$. We conclude that the rate of growth of the deformation will become significant for overburden thickness from one to five times the thickness of the layer. The corresponding dominant wavelength will be in the order of 10 to 20 times the salt layer thickness. This result appears to be compatible with the observed range of distances between initial salt structures.

\section{c) Dependence on the relative density contrast}

It has been found that the dominant wavelength is quite insensitive to changes in $\Delta \rho / \rho_{1}$. However, as can be expected, $t_{c}$ is extremely sensitive to such changes. For large $h_{1} / h_{2}$ an accurate estimate for the rate of amplification of the dominant wavelength can be made by assuming that $\sigma$ remains constant for varying $\Delta \rho / \rho_{1}$. This is brought out in Table 3 , in which $\sigma_{\min }$ is tabulated for various values of the parameters $\Delta \rho / \rho_{1}$ and $h_{1} / h_{2}$.

Table 3 Dependence of $\sigma_{\min }$ on $\Delta \rho / \rho_{1}$ and $h_{1} / h_{2}$
for case $\eta_{1} / \eta_{2}=1,000$

\begin{tabular}{l|cccc}
\hline \multirow{2}{*}{$\Delta \rho / \rho_{1}$} & \multicolumn{4}{|c}{$h_{1} / h_{2}$} \\
\cline { 2 - 5 } & 0.1 & 1 & 5 & 20 \\
\hline 0.01 & 39.6 & 4.35 & 1.00 & 0.69 \\
0.1 & 36.1 & 3.68 & 0.95 & 0.69 \\
0.2 & 32.1 & 3.29 & 0.90 & 0.69 \\
\hline
\end{tabular}

The characteristic times $t_{c}$ are given by equation (19), which shows that $t_{c}$ is proportional to $\sigma_{\min } /\left(\rho_{1}-\rho_{2}\right)$. Since for thickness ratios in excess of $5, \sigma_{\min }$ is insensitive to changes in the relative density contrast, $t_{c}$ is inversely proportional to the density contrast.

\section{d) Surface submerged under water}

The case in which a fluid of density $\rho_{f}$ lies above the free surface is included in the analysis. In this case, instead of a density contrast $\rho_{1}$ at the surface, we have a contrast $\rho_{1}-\rho_{f}$. This means that in the second equation (11) the density $\rho_{1}$ must be replaced by $\rho_{1}-\rho_{f}$, and the relative density ratio becomes $\Delta \rho /\left(\rho_{1}-\rho_{f}\right)$. Becouse the value of $\sigma_{\min }$, as shown by Table 3 , is insensitive 
to the relative density ratio, the characteristic times $t_{c}$ will not be appreciably changed by a layer of water overlying the top layer. Similarly the dominant wavelength, which is also insensitive to changes in $\Delta \rho / \rho_{1}$, will hardly be changed.

\section{e) Infuence of a nonrigid base}

Finally some remarks can be made concerning the influence of a nonrigid base. Biot (1963b) has analyzed the case of a single layer of viscosity $\eta_{1}$ between two identical infinite half-spaces of viscosity $\eta_{2}$. We shall compare this result with the case in which the bottom half-space is replaced by a rigid support. As already pointed out, this last case is obtained by putting $h_{1}=\infty$ in the previous analysis. The value of $\sigma$ versus $\gamma_{2}$ is plotted in Figure 5 for a rigid base (dashed curves) and for a viscous base (solid curves). Nonrigidity of the basement does not affect results significantly for $\eta_{1} / \eta_{3}>100$. The increase in dominant wavelength is affected by a factor less than 1.4. The characteristic time is slightly shorter for the viscous base, but for $\eta_{1} / \eta_{2}>100$, this effect is not more than about 10 percent.

\section{INSTABILITY WITH REDISTRIBUTION AT THE FREE SURFACE}

In the preceding discussion it was assumed that the overburden has a free surface which deforms into a corrugated surface of sinusoidal shape and an increasing amplitude as the unstable deforma- tion proceeds. It is of some interest to investigate what happens if the surface of the overburden remains flat. It is assumed that this flatness is obtained by any high spot on the upper surface being scraped off and the scraped-away material being deposited in the adjacent low spots. In this process the average thickness of the overburden remains the same. Such a procedure corresponds more or less to erosion of the upper surface by various geologic agents. We shall term it "redistribution." It should be noted that this surface condition is completely different from the condition by which the upper surface is kept flat by means of a weightless rigid plate.

Redistribution is simply accounted for by omitting the term $\rho_{1} g V_{1}$ in the second of equations (11). If we assume that the forces per unit area acting on the upper free surface are proportional to the height of the column of material added or removed, the contribution of gravity to $T_{v y}$ in equation (7), which is $\rho_{1} g V_{1}$, is exactly balanced by the force due to the redistribution, which is $-\rho_{1} g V_{1}$ (Figure 6). Another way of looking at this problem is to consider equations (11) as defining the velocities $\partial U_{1} / \partial t, \partial V_{1} / \partial t$, etc., as functions of the instantaneous values of $V_{1}$ and $V_{2 .}$ For "redistribution" we put $V_{1}$ equal to zero, at all instants, with the top surface remaining free. In terms of the analog model, redistribution implies the omission of the stabilizing surface force $\rho_{1} g V_{1}$ at the upper free surface.
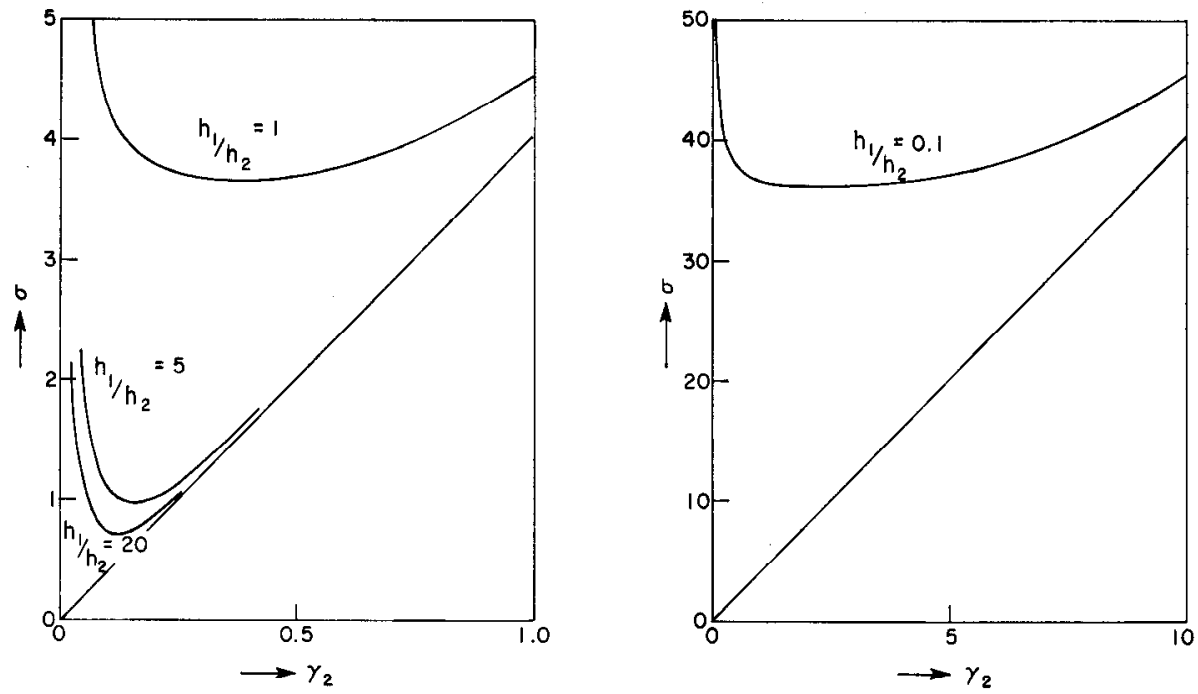

FIG. 4. $\sigma$ versus $\gamma_{2}$ for $\eta_{1} / \eta_{2}=1,000, \Delta \rho / \rho_{1}=0.1$, and varying thickness ratio. 


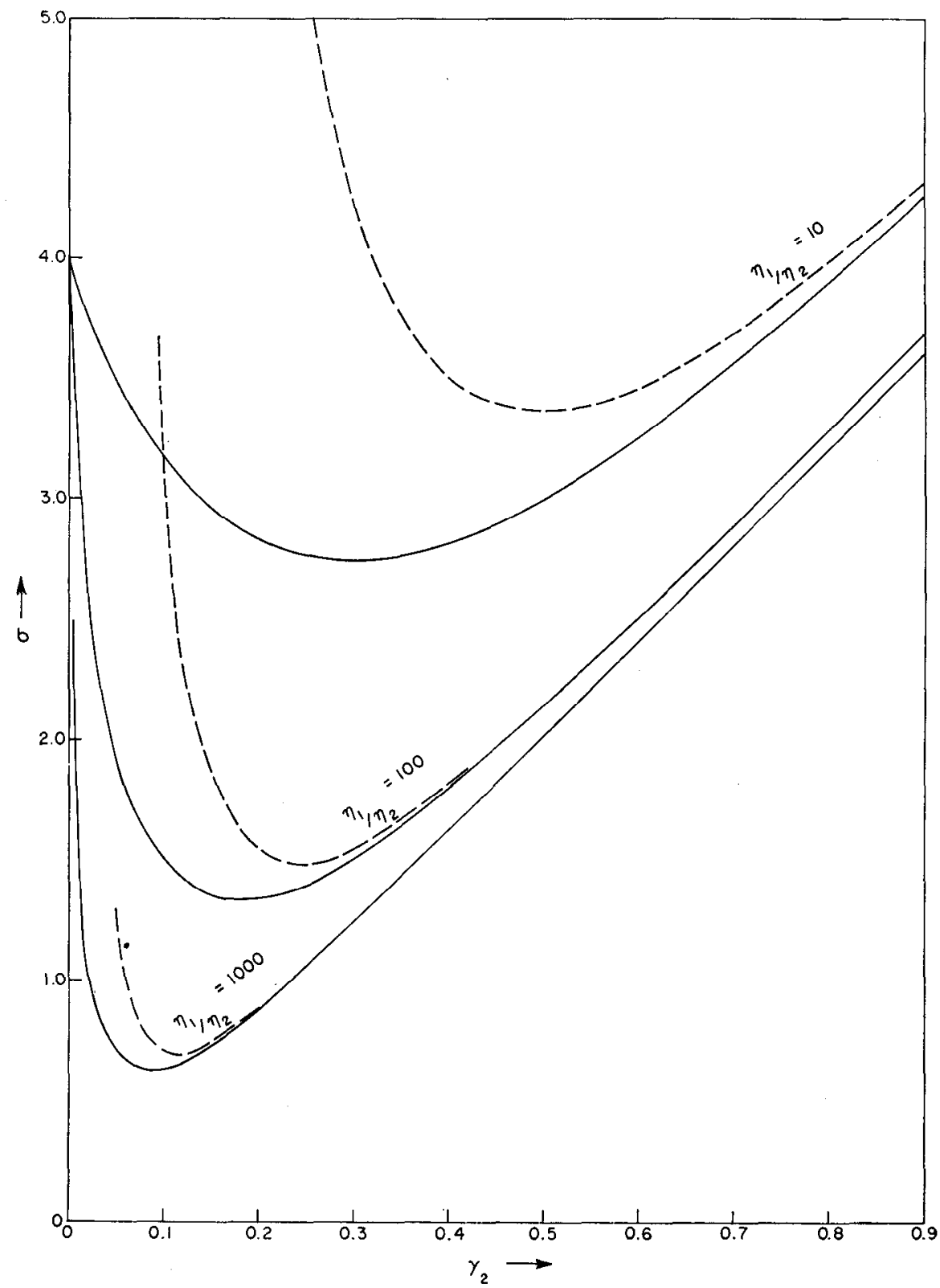

FIG. 5. Stability curves for infinite overburden and rigid base (dashed) and infinite overburden and viscous base (solid).

The stability equation similar to (15) is now a linear instead of a quadratic one,

$$
a^{\prime} \sigma+b^{\prime}=0
$$

where $a^{\prime}$ and $b^{\prime}$ are, as before, functions of the three nondimensional parameters. In the same manner as before, $\sigma$ can be plotted versus $\gamma_{2}$. Figure 7 shows such curves for varying $\eta_{1} / \eta_{2}$ but constant $h_{1} / h_{2}(=1)$ and relative density contrast $\Delta \rho / \rho_{1}(=0.1)$. For short wavelengths the curves of Figures 3 and 7 are almost identical, but for wavelengths of the order of and longer than the dominant wavelength, there is considerable 


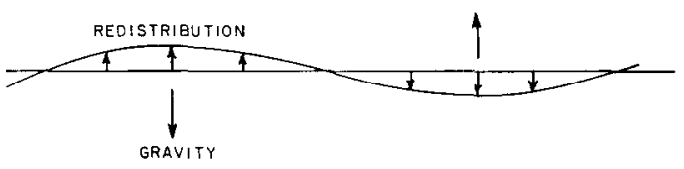

Fig. 6. Force applied to upper surface, equivalent to "redistribution."

differcnce, consisting of a longer dominant wave length and, most important, a quite significant reduction in time $t_{c}$. The latter is not surprising. The extra surface force attendant with the process of "redistribution" acts exactly in a way to accelerate the instability. In Table 4 the ratio $L_{d} / h_{2}$ and the time $t_{c}$ are tabulated for the case shown in Figure 7, computed for values given in

\section{Table 4}

Redistribution case $h_{1} / h_{2}=1$; $\Delta \rho / \rho_{1}=0.1$

\begin{tabular}{rrl}
\hline$\eta_{1} / \eta_{2}$ & $L_{d} / h_{2}$ & $t_{c}$ (years) \\
\hline 1 & 3.92 & $0.007 \times 10^{6}$ \\
10 & 5.76 & $0.012 \times 10^{6}$ \\
100 & 8.37 & $0.026 \times 10^{6}$ \\
1,000 & 12.20 & $0.059 \times 10^{6}$
\end{tabular}

equation (20). As shown by comparison with Table 1 , the reduction in $t_{c}$ is particularly obvious for high viscosity ratios. It is to be expected that the greatest changes in $t_{c}$ will occur for very thin upper layers. This is shown by comparing Table 2 with Table 5 , in which the ratio $I_{d} / h_{2}$ and the time $t_{c}$ are tabulated for constant $\eta_{1} / \eta_{2}(=1,000)$ and $\Delta \rho / \rho_{1}(=0.1)$ but varying $h_{1} / h_{2}$. This com-

Table 5

Redistribution case $\eta_{1} / \eta_{2}=1,000$; $\Delta \rho / \rho_{1}=0.1$

\begin{tabular}{ccc}
\hline$h_{1} / h_{2}$ & $L_{d} / h_{2}$ & $t_{c}$ (years) \\
\hline 0.1 & 4.03 & $0.009 \times 10^{6}$ \\
1.0 & 12.20 & $0.059 \times 10^{6}$ \\
5 & 26.18 & $0.268 \times 10^{6}$ \\
20 & 28.23 & $0.622 \times 10^{6}$ \\
$\infty$ & 27.6 & $0.63 \times 10^{6}$ \\
\hline
\end{tabular}

parison shows that redistribution does not significantly alter the dominant wavelength. However, for this overburden the characteristic time is shortened drastically. The dependence of $t_{c}$ on the overburden thickness is also completely reversed. With redistribution it increases toward a limiting value as the overburden thickness tends to infinity.

\section{INSTABILITY WITH TIME-DEPENDENT THICKNESS AND COMPACTION OF OVERBURDEN}

So far we have considered only cases in which all parameters remained constant. Actually these conditions are not fulfilled. Compaction of sediments during geologic time will increase the density contrast $\Delta \rho$, and lithification of the overburden will increase its viscosity. Of special relevance is the case in which the thickness of the overburden increases during development of the instability. Then the dominant wavelength is no longer constant but increases with increasing thickness of overburden. Changes in growth rates of individual wavelengths with increasing thickness of overburden, and the consequent shift in dominant wavelength, are brought out in Figure 8 , in which the quantity $1 / \sigma$, proportional to $p$ according to equation (17), versus the thickness ratio $h_{1} / h_{2}$ for a number of wavelengths in the case $\Delta \rho / \rho_{1}=1 / 10$ and $\eta_{1} / \eta_{2}=1,000$ is shown graphically.

The figure shows that initially, i.e., for small thickness of overburden, short wavelengths grow

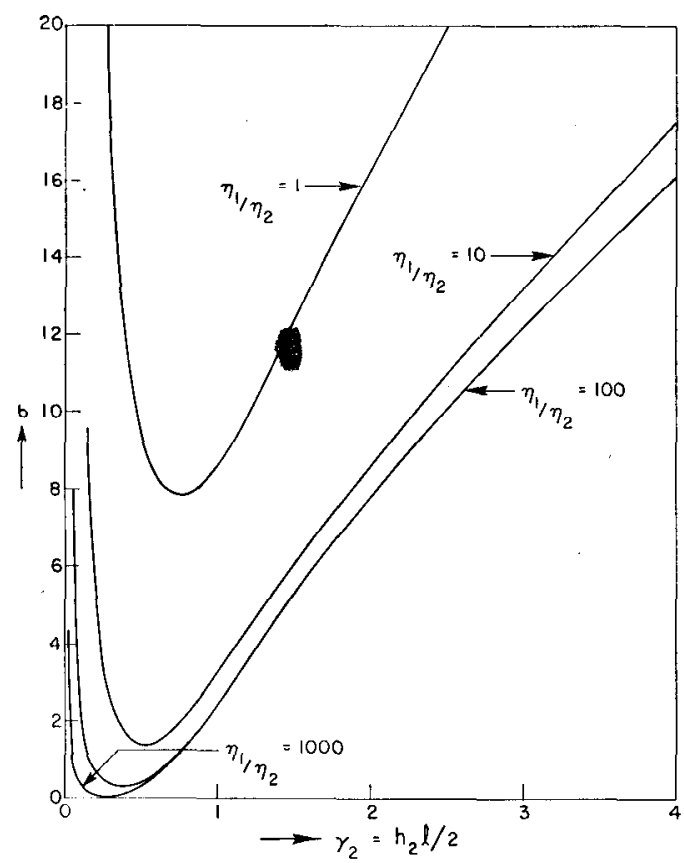

FIG. 7. Redistribution. $\sigma$ versus $\gamma_{2}$ for $h_{1} / h_{2}=1, \Delta \rho / \rho_{1}=0.1$. 
faster than long ones and soon reach a constant rate. With increasing thickness of overburden, the rate of growth for longer wavelengthis continues to increase, whereas it stops for the shorter wavelengths. This results in a crossover of curves in Figure 8 as, for example, the curves for $L=10 \mathrm{~km}$ and $L=30 \mathrm{~km}$. For very long wavelengths $(L>30 \mathrm{~km})$, the curves will rise slowly and do not reach as high a value of $1 / \sigma$ as those for $L$ in the neighborhood of the dominant wavelength. The fastest growing wavelength at any value of $h_{1} / h_{2}$ is the one for which $1 / \sigma$ has the maximum value; it is clear from Figure 8 that this wavelength shifts to higher values with increasing $h_{1} / h_{2}$ and approaches some maximum constant value for the dominant wavelength.

In this section we shall consider in some detail the influence of changes in density $\rho_{1}$ and the thickness ratio during the instability. Let it be assumed that $h_{1}$ increases linearly with time and that $h_{2}\left(=10^{5} \mathrm{~cm}\right)$ remains constant:

$$
\frac{h_{1}}{h_{2}}=\frac{t}{t_{0}},
$$

where $t_{o}$ represents the time in which the overburden reaches a thickncss cqual to that of the salt layer. The thickness ratio is then a measure of time. At any moment the overburden, whose density varies in the $y$ dircetion, can be thought to be composed of a large number of layers, each with a density $\rho_{i}$. It was shown in Section 2 that the cffect of gravity is analogous to the case of a weightless layer with the addition of an interfacial force of magnitude $\left(\rho^{\prime}-\rho\right) g V$ per unit area and acting downward. Each thin layer contributes such a force. The idea is to replace the total overburden by a single layer having an "effective" density $\rho_{e}$ so that its behavior approximates that of the sum of the discrete layers.

It was shown by Biot (1963b) that equations (11) corresponding to a multilayered case could be written formally as

$$
\frac{\partial T}{\partial U_{i}}=0, \quad \frac{\partial(T+G)}{\partial V_{i}}=0,
$$

in which the function $T$ is an invariant corresponding to the potential energy of the whole system, and $G$ is an invariant from which the interfacial force due to gravity is derived. $G$ is given for an $n$-layered medium by

$$
G=\frac{1}{2 l} \sum_{i=0}^{n}\left(\rho_{i+1}-\rho_{i}\right) g V_{i+1}^{2} .
$$

The overburden is now replaced by a single layer of effective density $\rho_{e}$ so that $G$ remains unchanged; that is,

$$
G=\frac{1}{2 l}\left[\rho_{e} g V_{1}^{2}-\left(\rho_{e}-\rho_{2}\right) g V_{2}^{2}\right] .
$$

Equating (24) and (25), one obtains

$$
\begin{aligned}
-\rho_{e} g V_{1}^{2}+\left(\rho_{e}-\rho_{2}\right) g V_{2}{ }^{2} & \\
& =\sum_{i=0}^{n}\left(\rho_{i}-\rho_{i+1}\right) g V_{i+1}^{2} .
\end{aligned}
$$

If we denote the density of the overburden by $\rho_{t}$ at the top and by $\rho_{n}$ at the bottom, we can write in the limit, when $n \rightarrow \infty$,

$$
\begin{gathered}
-\rho_{e} V_{1}{ }^{2}+\left(\rho_{e}-\rho_{2}\right) V_{2}{ }^{2}=-\rho_{1} V_{1}{ }^{2}+\left(\rho_{n}-\rho_{2}\right) V_{2}{ }^{2} \\
+\int_{-h_{\imath}}^{0} \frac{\partial \rho}{\partial y} V^{2}(y) d y
\end{gathered}
$$

in which $\partial \rho / \partial y<0$ for density increasing with depth. In the evaluation of $\rho_{e}$ from equation (27) we use the relation between density and depth of sediments determined by Nettleton (1934) for the Gulf Coast region. This relation is shown by curve $A$ in Figure 9 and can be approximated by the function

$$
\rho(y)=2.49-0.59 e^{\alpha y}-a y e^{\beta y},
$$

in which

$$
\begin{aligned}
& \alpha=0.59 \times 10^{-5} \mathrm{~cm}^{-1}, \\
& \beta=1.6 \times 10^{-5} \mathrm{~cm}^{-1}, \\
& a=5 \times 10^{-6} \mathrm{~g} / \mathrm{cm}^{2},
\end{aligned}
$$

and $-y$ is the depth expressed in $\mathrm{cm}$. The curve representing equation (28) is shown as $B$ in Figure 9.

The amplitude of the displacement at any depth $y, V(y)$, can be expressed as a function of $y, V_{1}$, and $V_{2}$ by means of the solution employed in any one layer of the medium which is of the type

$V(y)=k_{1} e^{l y}+k_{2} e^{-l y}+k_{3} y e^{l y}+k_{4} y e^{-l y}$. 


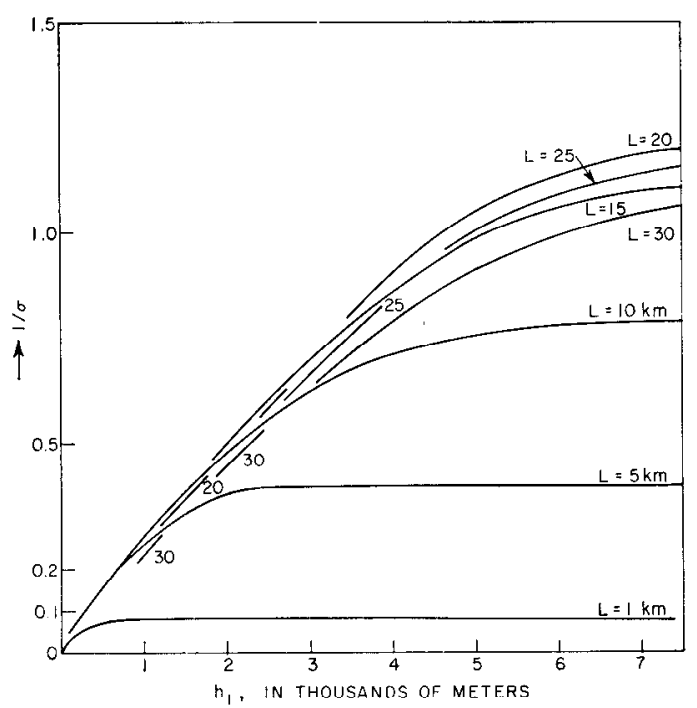

Fig. 8. Instantaneous rate of amplification of various wavelengths $L$ as function of thickness of overburden. No redistribution, $\Delta \rho / \rho_{1}=0.1, \eta_{1} / \eta_{2}=1,000$.

The constants $k_{i}$ are determined by the conditions

1) $V(y)=V_{1}$ for $y=0$,

2) $V(y)=V_{2}$ for $y=-h_{1}$, and

3) The incremental stresses $T_{x y}$ and $T_{y y}$ are zero at the free surface $y=0$.

We find that the integration constants $k_{i}$ can be simply expressed as linear functions of $V_{1}$ and $V_{2}$. Noting that equation (27) is homogeneous in $V_{1}$ and $V_{2}$, we can eliminate these amplitudes once the ratio $V_{1} / V_{2}$ is known. In principle this is possible by means of equation (11). However, a simpler result is obtained by putting $V_{1}=0$. This is permissible because the ratio $V_{1} / V_{2}$ is quite small, as can be checked for various wavelengths and the parameters $\eta_{1} / \eta_{2}=1,000, \Delta \rho / \rho_{1}=0.1$ by means of equation (11). Consideration of the analog model shows why this should be so. At the bottom of the overburden the rate of energy input by the interfacial force into the system is proportional to

$$
\left(\rho_{1}-\rho_{2}\right) g V_{2} d V_{2},
$$

and at the free surface it is proportional to

$$
-\rho_{1} g V_{1} d V_{1}
$$

Since energy is dissipated in the system,

$$
\left(\rho_{1}-\rho_{2}\right) g V_{2} d V_{2}>\rho_{1} g V_{1} d V_{1} ;
$$

thus

$$
V_{2}>V_{1} \text {. }
$$

For $V_{1}=0$ we obtain

$$
V(y)=\frac{-l y \cosh l y}{2 \gamma_{1} \cosh 2 \gamma_{1}} V_{2} .
$$

The effective density is therefore given by

$$
\rho_{e}=\rho_{n}+\int_{-h_{1}}^{0} \frac{y^{2} \cosh ^{2} l y}{h_{1}^{2} \cosh ^{2} l h_{1}} \frac{\partial \rho}{\partial y} d y .
$$

Equation (32) shows that for short wavelengths $(l \rightarrow \infty) \rho_{e}$ tends to $\rho_{n}$, but that for long wave-

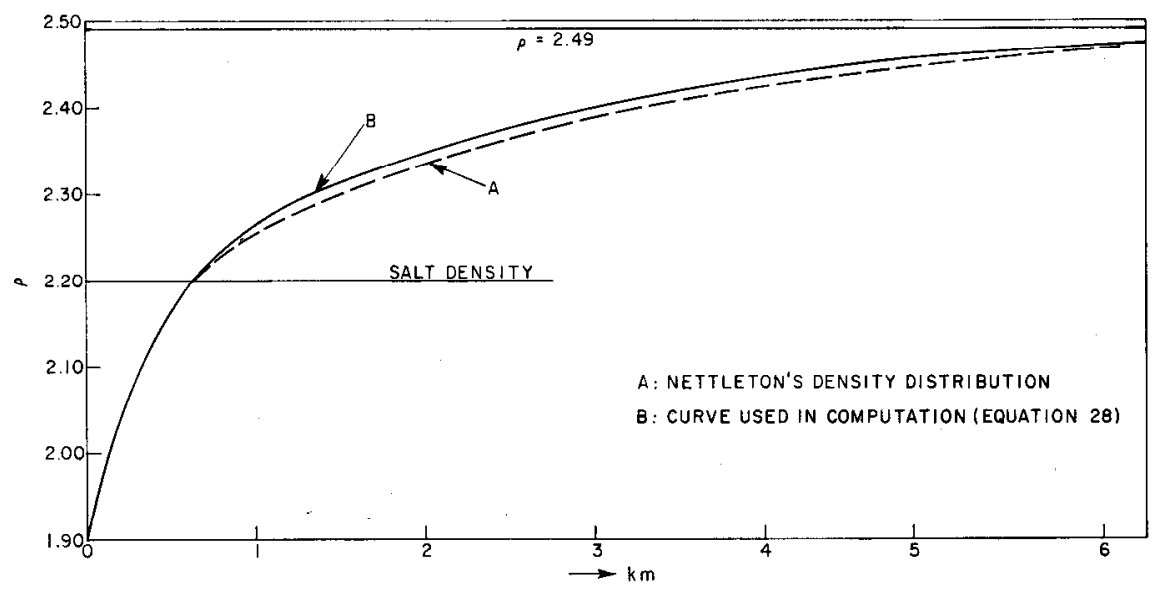

FIG. 9. Density-depth relation for Gulf Coast sediments. 


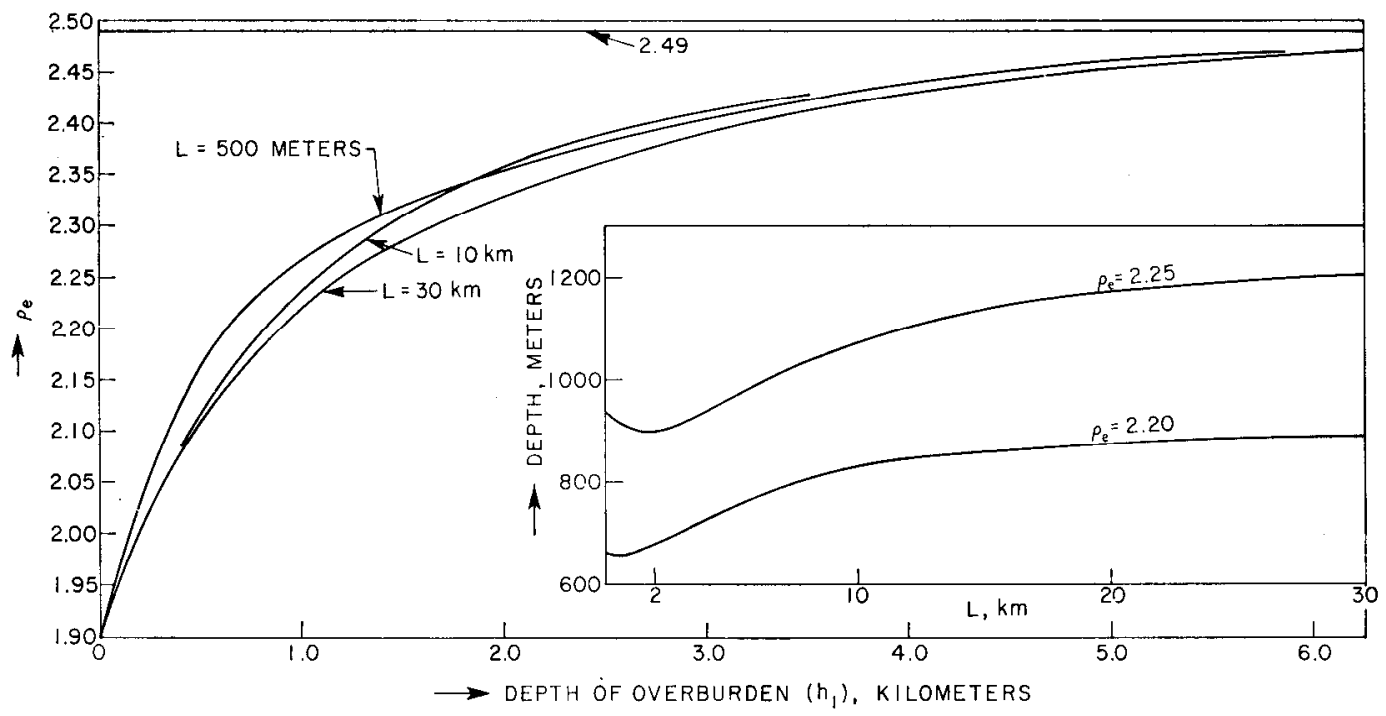

FIG. 10. Effective density for $L=\frac{1}{2}, 10$, and $30 \mathrm{~km}$. In insert is shown the depth of overburden at which a given effective density is reached for wavelengths up to $30 \mathrm{~km}$.

lengths $\rho_{e}<\rho_{n}$. In Figure 10 the effective density is shown for various wavelengths as a function of $h_{1} / h_{2}$. The instability will start as soon as the effective density $\rho_{e}$ exceeds $\rho_{2}$, the salt density.

An estimate of the time dependence of the instability of the salt can now be made as follows. First the depth at which $\rho_{e}$ exceeds the density of the salt is computed. This depth is slightly dependent on the wavelength, as shown in the insert of Figure 10 . The time $t_{L}$, which we call delay time, corresponding to this depth depends on the rate of sedimentation. Various geologists (Murray, 1952; Shepard, 1953; Kay, 1955) have made estimates of the following magnitudes:

$$
\begin{aligned}
& 50 \mathrm{~cm} / 10^{3} \text { years (fast), } \\
& 20 \mathrm{~cm} / 10^{3} \text { years (intermediate), } \\
& 10 \mathrm{~cm} / 10^{3} \text { years (slow). }
\end{aligned}
$$

In equation (22) these rates correspond (for $h_{2}=10^{5} \mathrm{~cm}$ ) to $t_{0}=6.3 \times 10^{13}, 1.6 \times 10^{14}$, and $3.15 \times 10^{14} \mathrm{sec}$, respectively.

From the instantaneous amplification rates, as shown in Figure $8, p(L, t)$ can be estimated as a function of depth of overburden and hence of time $t$ and wavelength $L$. The amplification $A$ at time $t$ of a specified wavelength $L$ is given by

$$
A=\exp \left[\int_{t_{L}}^{t} p(L, t) d l\right]
$$

that is, essentially, by the area underneath the curves of Figure 8 in which the horizontal scale is converted into a time scale by means of the given rates (33). The crossover of the curves in Figure 8 indicates that the time required to obtain a given amplification reaches a minimum for some wavelength close to $20 \mathrm{~km}$.

The integrand in equation (34) can be written as

$$
p(L, t) d t=\frac{g h_{2} t_{0}}{\eta_{1}}\left(\rho_{e}-\rho_{2}\right) \frac{1}{\sigma} d\left(\frac{h_{1}}{h_{2}}\right) .
$$

An estimate of the amplification time $\ell$ is made by assuming that $1 / \sigma$ does not vary with the density contrast $\rho_{e}-\rho_{2}$.

Then since $\rho_{e}-\rho_{2}$ and $1 / \sigma$ are known as functions of $h_{1} / h_{2}, A$ can be estimated graphically by means of equation (35). The result for the intermediate rates of sedimentation (33) is shown in Figure 11.

The characteristic time $t_{c}$ corresponds to the curve for which $A=10^{3}$. The wavelength $L$ which is at some time $t$ amplified most will, in general, not be the wavelength which at that moment is being amplificd at the fastest rate. Hence, we must make a distinction between two types of dominant wavelength-the instantaneous dominant wavelength and the physically dominant wavelength, that is, the wavelength amplified the 


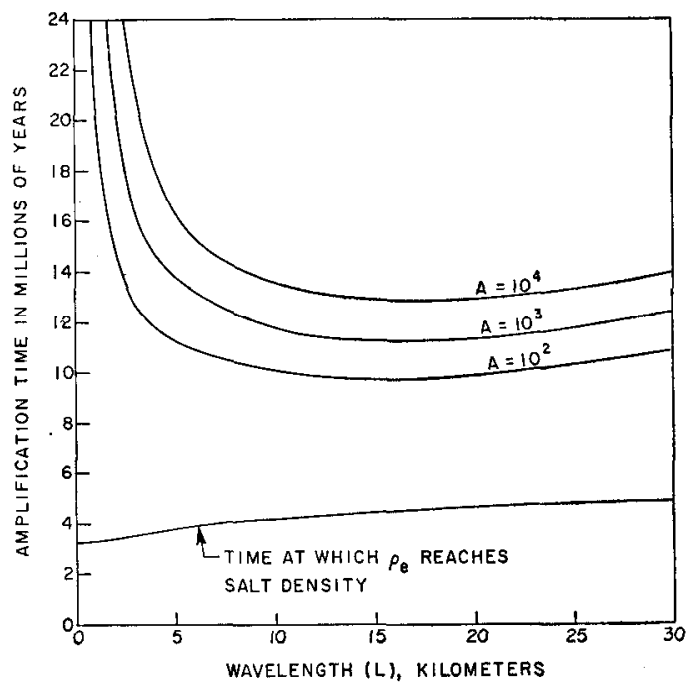

Frg. 11. Time $t$ required to obtain a given amplification factor $A$ under conditions of variable compaction and thickness of overburden (rate of sedimentation $20 \mathrm{~cm}$ per $10^{3}$ years). The curve for $A=10^{3}$ corresponds to the characteristic time $t_{c}$.

most at the time $t$. It is the latter which is indicated in Figure 11.

The rate of sedimentation makes some difference in the final Figure 11. If this rate is small, the time $t_{c}$ will be larger and the dominant wavelength will be somewhat shorter than indicated in Figure 11. If the rate of sedimentation is fast, the observed dominant wavelength will be somewhat longer than is indicated, and the times will be shorter. Whatever the rate of sedimentation may be, the time history can be described as follows: First there is a period in which the effective density of the sediment is increased to that of the salt. Only then does the instability set in. This time is shorter for fast rates than for slow rates. It is probable that in many salt basins the initial rates are relatively slow, and thus several million years are needed for the instability to start. After that initial stage, the sediment density slowly builds up. During this time the density contrast is small, and the dominant wavelength is relatively short, but the rate of growth of any wavelength also remains small. This period might be described as the "incubation period" of the salt structure. Finally, in the third period enough sediment has accumulated to create sufficient density contrast, the dominant wavelength has increased to a wavelength of the order of $15-20 \mathrm{~km}$, and the accompanying rates have grown so large that the instability starts growing rapidly. It is now that the salt structure takes shape and finds its expression in a series of well-defined undulations of the overburden-salt interface. In Figure 11 a tenfold increase in amplitude for the dominant wavelength is attained in the order of only one million years, which shows that all the important stratigraphical relationships observed around the emerging salt structure possibly originated in a relatively short time as compared with the total lifetime of the instability. The curves in Figure 11 are based on the assumption of a uniform rate of sedimentation throughout the basin. This assumption is only very approximately true. The precise time dependence of sedimentation rates throughout a given basin has an important bearing on the growth of certain groups of structures and the relative quiescence of others in the same basin. However, this subject is beyond the scope of this paper.

Figure 11 shows a rather broad minimum, which means that the amplification is not very selective. Initial perturbations on the overburdensalt interface are probably of short extent. This means that they are not much amplified, but that in the presently observed interface only wavelengths longer than $10 \mathrm{~km}$ are likely to be observed. Moreover the shape of the curves of Figure 11 is not particularly sensitive to the values of the initial thickness of the salt $\left(h_{2}\right)$, the viscosity ratio between overburden and salt, and the relative density contrast. These values, rather, determine the length of the "incubation time" of the structure. This may explain the somewhat uniform appearance of many salt structures in various basins in the world.

We must also emphasize that the present theory covers only the initial phase of the growth of salt structures. The vertical ascent of salt in pillars or "chimney"-like structures, so well known in the Gulf Coast Basin of the United States of America, represents a later phase in the process of instability, which we do not discuss here.

\section{ACKNOWLEDGMENT}

The authors are indebted to $\mathbf{M}$. King Hubbert for many fruitful discussions of the geological aspects of the present theory.

\section{REFERENCES}

Arrhenius, S., 1912, Zur Physik der Salzlagerstätten: Medd. Vetenskaps akad. Nobelinst, v. 2, p. 1-25.

Biot, M. A., 1961, Theory of folding of stratified 
viscoelastic media and its implications in tectonics and orogenesis: Bull. Geol. Soc. Amer., v. 72, p. 15951620 .

1963a, Theory of stability of multilayered continua in finite anisotropic elasticity: J. Franklin Inst., v. 276 , p. $128-153$.

$1963 \mathrm{~b}$, Stability of multilayered continua including the effect of gravity and viscoelasticity: J. Franklin Inst., v. 276, p. 231-252.

Hubbert, M. King, 1937, Theory of scale models as applied to the study of geologic structures: Bull. Geol. Soc. Amer., v. 48, p. 1459-1520.

Kay, M., 1955, Sediments and subsidence through time, in The crust of the earth: Geol. Soc. Amer., Special Paper No. 62, p. 675-684.

Murray, G. E., 1952, Sedimentary volumes in Gulf
Coastal plain of the United States and Mexico, Foreword and summary: Bull. Geol. Soc. Amer., v. 63, p. 1157.

Nettleton, L. L., 1934, Fluid mechanics of salt domes: Bull. A.A.P.G., v. 18, p. 175-204.

Odé, H., 1962, Review of mechanical properties of salt relating to salt dome genesis: Shell $F$ \& $P$ Research Pub. 344, Paper presented before Intern. Conf, on Saline Deposits, Div. Earth Sci., Natl. Res. Coun., Houston, Texas, Nov. 2-23.

Parker, T. J., and McDowell, A. N., 1955, Model studies of salt dome tectonics: Bull A.A.P.G., v. 39, p. 2384-2471.

Shepard, F. P., 1953, Sedimentation rates in Texas estuaries and lagoons: Bull. A.A.P.G., v. 37, p. 1919 1934. 